\title{
ENERGY TRANSFER IN CONTACT BINARIES
}

\author{
A. P. MOSES and R. C. SMITH \\ Astronomy Centre, University of Sussex, Falmer, Brighton, England
}

\begin{abstract}
The anomalous mass-luminosity relation for the components of a contact binary system is usually explained by postulating strong energy transfer from the primary to the secondary. It has been assumed that the transfer occurs in the common convective envelope surrounding the two stars, but so far the only attempt at a model for the energy transfer has been the sideways convection model of Hazlehurst and Meyer-Hofmeister (1973), which assumes a large-scale circulation of material between the two components.

Any detailed discussion of the dynamics in the common envelope must take account of the predominantly vertical motions associated with normal thermal convection, of Coriolis forces and of viscosity. We have constructed an approximate model for the horizontal transfer of energy between the two components, using a mixing-length approach and taking all three factors into account. The major factors are the vertical convection and the Coriolis forces, which together prevent a large-scale circulation of the type proposed by Hazlehurst and Meyer-Hofmeister. Instead, the flow breaks up into smallscale eddies whose horizontal scale is determined by the interaction of convection, Coriolis forces and viscosity. This has the important qualitative consequence that horizontal energy transfer will occur only if the mean horizontal pressure gradient between the two stars exceeds a certain minimum value. This condition can easily be satisfied in the adiabatic zone of the envelope, but may be an important restriction in the superadiabatic zone.

Using our model, we were able to estimate the entropy difference between components which is required to transfer enough energy to explain the observed massluminosity relation. We found that equal entropy models are possible only if the contact is deep. Unequal entropy models are possible for any degree of contact, so long as the contact extends down as far as the adiabatic zone. If, as has been suggested, the depth of contact increases during evolution then zero-age models must have shallow contact and hence unequal entropies. Deep contact equal entropy models would then form as a result of evolution.
\end{abstract}

A difficulty is that in our model insufficient energy transfer can occur in the superadiabatic zone to produce WUMa light curves for the unequal entropy models. This may mean that further work is needed on the exact surface conditions in these stars.

\section{Reference}

Hazlehurst, J. and Meyer-Hofmeister, E.: 1973, Astron. Astrophys. 24, 379.

P. Eggleton et al. (eds.), Structure and Evolution of Close Binary Systems, 333. All Rights Reserved. Copyright $\odot 1976$ by the IAU. 